\title{
X-ray Interferometry Development at the Advanced Photon Source*
}

\author{
K. Fezzaa and W.-K. Lee \\ Advanced Photon Source, Argonne National Laboratory \\ Argonne, IL 60439
}
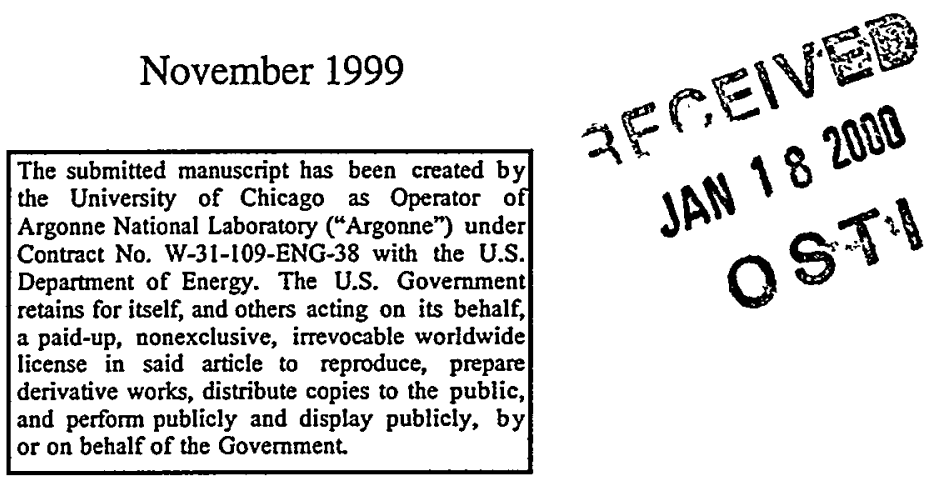

To be published in the Proceedings of the $11^{\text {th }}$ U.S. National Synchrotron Radiation Instrumentation Conference (SRI'99), Stanford, CA, October 13-15, 1999.

*This work is supported by the U.S. Department of Energy, Basic Energy Sciences, Office of Science, under contract \#W-31-109-ENG-38. 


\section{DISCLAIMER}

This report was prepared as an account of work sponsored by an agency of the United States Government. Neither the United States Government nor any agency thereof, nor any of their employees, make any warranty, express or implied, or assumes any legal liability or responsibility for the accuracy, completeness, or usefulness of any information, apparatus, product, or process disclosed, or represents that its use would not infringe privately owned rights. Reference herein to any specific commercial product, process, or service by trade name, trademark, manufacturer, or otherwise does not necessarily constitute or imply its endorsement, recommendation, or favoring by the United States Government or any agency thereof. The views and opinions of authors expressed herein do not necessarily state or reflect those of the United States Government or any agency thereof. 


\section{DISCLAIMER}

Portions of this document may be illegible in electronic image products. Images are produced from the best available original document. 


\title{
X-ray Interferometry Development at the Advanced Photon Source
}

\author{
K. Fezzaa and W-K. Lee \\ Advanced Photon Source, Argonne National Laboratory, Illinois, 60439
}

\begin{abstract}
In this paper, we report initial test results of a four-bounce Bragg reflection Xray interferometer at $7.46 \mathrm{keV}$ and, for the first time to our knowledge, at the higher energy of $14.91 \mathrm{keV}$ where the spectral acceptance is much smaller.
\end{abstract}

\section{INTRODUCTION}

The triple Laue (LLL) interferometer is one of the most used X-ray interferometers, be it in ultra-precision metrology ${ }^{1}$ or in imaging techniques ${ }^{2}$. The advantage of the LLL interferometer is that it can be used within a wide range of energy and is easy to manufacture when compared to other interferometer types. As a first attempt into $\mathrm{x}$-ray interferometry, we have tested a triple Laue interferometer and measured contrasts better than about $90 \%$ when sliding a plastic wedge into one of the two interfering beam paths.

However, the Laue transmission geometry has two drawbacks: (1) absorption losses in each of the blades, and (2) loss of spatial resolution due to the Borrman broadening in the last blade. These two disadvantages can be overcome by using all Bragg reflection interferometers.

One such design was proposed by Graeff and Bonse ${ }^{3}$. The main idea was to simultaneously excite two complementary (Si(440) and Si(404)) atomic planes in Bragg geometry to split the beam and gather them again after several Bragg reflections (Fig. 1). The built-in spectral window of such a dispersive device is extremely narrow (compared with the LLL) and it only works at a particular wavelength. This makes it relatively difficult to operate and puts stringent requirements on the crystal.

Graeff and Bonse ${ }^{3}$ first successfully tested this three-beam interferometer using an X-ray tube (near Ni $K \alpha_{2}, 48$ hour exposure time). Later, they tested it again at a synchrotron and obtained about $70 \%$ contrast with a wedge. The nominal wavelength for this interferometer is $\lambda=1.66276 \AA$, with a corresponding interferometer transmission range $\Delta \lambda / \lambda \sim 410^{-6}$ for $\pi$ polarization. The original Graeff-Bonse interferometer incorporated several weak links in the monolith so that one part of the monolith could be translated with respect to the other, thereby introducing micron-range path length differences between the two paths.

As a first attempt with this type of interferometer, we fabricated a simplified version with no movable parts. The crystal, shown in Figure 1, was made of a single block of high-quality float-zone silicon. 
We report in this paper the initial test results of this interferometer at $7.46 \mathrm{keV}$, as well as, for the first time to our knowledge, at the higher energy of $14.91 \mathrm{keV}$ (using the $\mathrm{Si}(880)$ and $\mathrm{Si}(808)$ reflections) where the spectral acceptance is about an order of magnitude smaller.
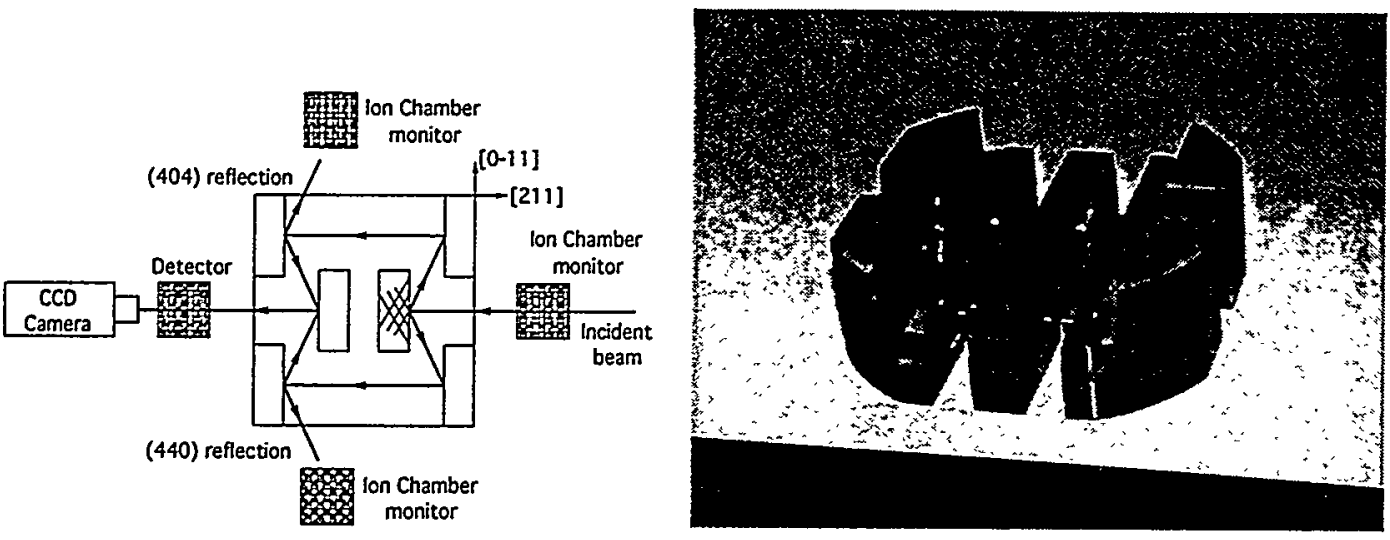

FIGURE 1. Four Bragg reflection (BBBB) interferometer geometry (left) and the actual silicon crystal (right).

\section{MEASUREMENTS AT $7.46 \mathrm{keV}$ - Si(440)/Si(404)}

The interferometer was tested on the SRI-CAT 1-ID beamline at the Advanced Photon Source (APS). A double-crystal monochromator, which diffracts vertically with $\mathrm{Si}(111)$ crystals, was upstream of the interferometer. The interferometer itself diffracts in the horizontal (electron orbit) plane and is housed in a Plexiglas box to minimize air-flow-induced instabilities. For an incoming photon rate from the monochromator of about $10^{13} \mathrm{ph} / \mathrm{s}$, the transmitted rate was about $10^{8}-10^{9} \mathrm{ph} / \mathrm{s}$.

Figure 2 shows the setup used to measure the contrast obtained through a 50 $\mu \mathrm{m}$ aperture in front of an ionization chamber detector, while sliding a $4^{\circ}$ plastic wedge in one arm of the interferometer. A thin piece of plastic attenuator in the other arm compensates for the absorption loss through the wedge.
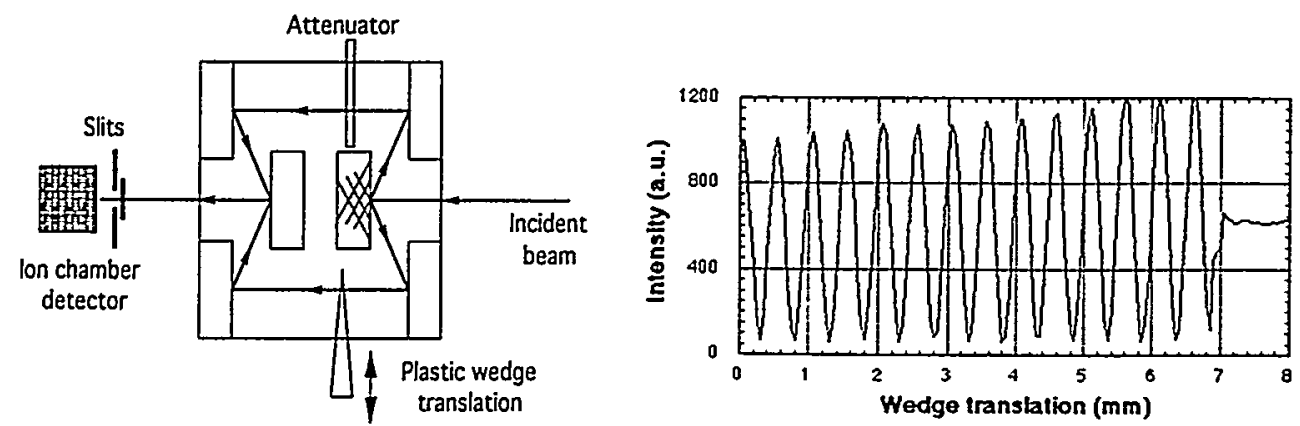

FIGURE 2. Wedge contrast setup top view (left) and the fringes obtained with the wedge (right). 
The measured contrast was about $93 \%$. The small increase in the contrast (Fig. 2) at larger wedge translation-where the wedge becomes thinner-is an absorption effect. The contrast reaches its peak when the two interfering beams have equal amplitudes.

The crystal contains residual strains, either from the fabrication process or slight imperfections in the silicon material itself. This results in Moire fringes in the transmitted beam, as shown in Figure 3. The interfringe distance indicates an angular mismatch of 2 arc seconds. The setup to take this picture is also shown in Figure 3.
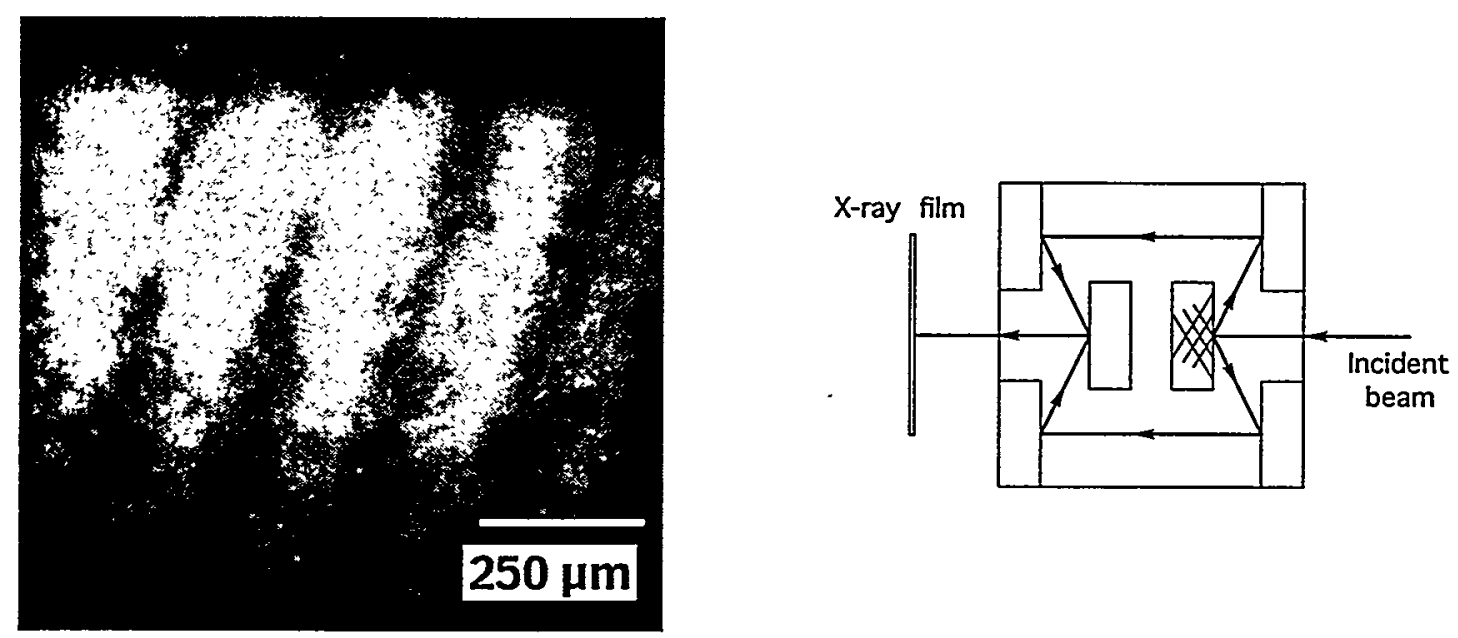

FIGURE 3. Moire fringes recorded on a Polaroid film at $7.46 \mathrm{keV}$ (left) and the setup used (right).

\section{PHASE CONTRAST IMAGING}

Using a nonabsorbing sample of animal cancer cells deposited on a thin plastic film, phase contrast images were obtained (Fig. 4). With minor changes in the setup, three methods of phase contrast imaging techniques ${ }^{2,4,5}$ were used. The arrows in the images point to the same details in the sample.

The first type of phase contrast image is a Fresnel (or propagation) image ${ }^{4}$, when the sample is downstream of the crystal (Fig. 4a) with the CCD camera at $30 \mathrm{~cm}$ from the sample. Here, the interferometer is irrelevant, and only the lateral coherence of the beam matters. Fresnel images are sensitive to sharp phase jumps, and the edges are well enhanced. These enhancements come from the interference between the distorted and undistorted wave-fronts (assuming a plane wave) at the edge of a phase object.

The second type of phase contrast image is an angle-resolved or differential phase image 5 . The sample is placed in one arm of the interferometer, while the other arm is blocked (Fig. 4b). A phase gradient across a wave-front is equivalent to a change in the local propagation direction of the wave ${ }^{6}$. Thus, using a crystal reflection as an angular analyzer, one can detect the small angular deviations of the beam and, thus, detect the edges of the phase object (or location of the phase jumps). In the 
interfiorometer, the reflections after the sample act as such angle analyzers. The contrast in the cells is in the horizontal plane, which is the diffraction plane of the setup.
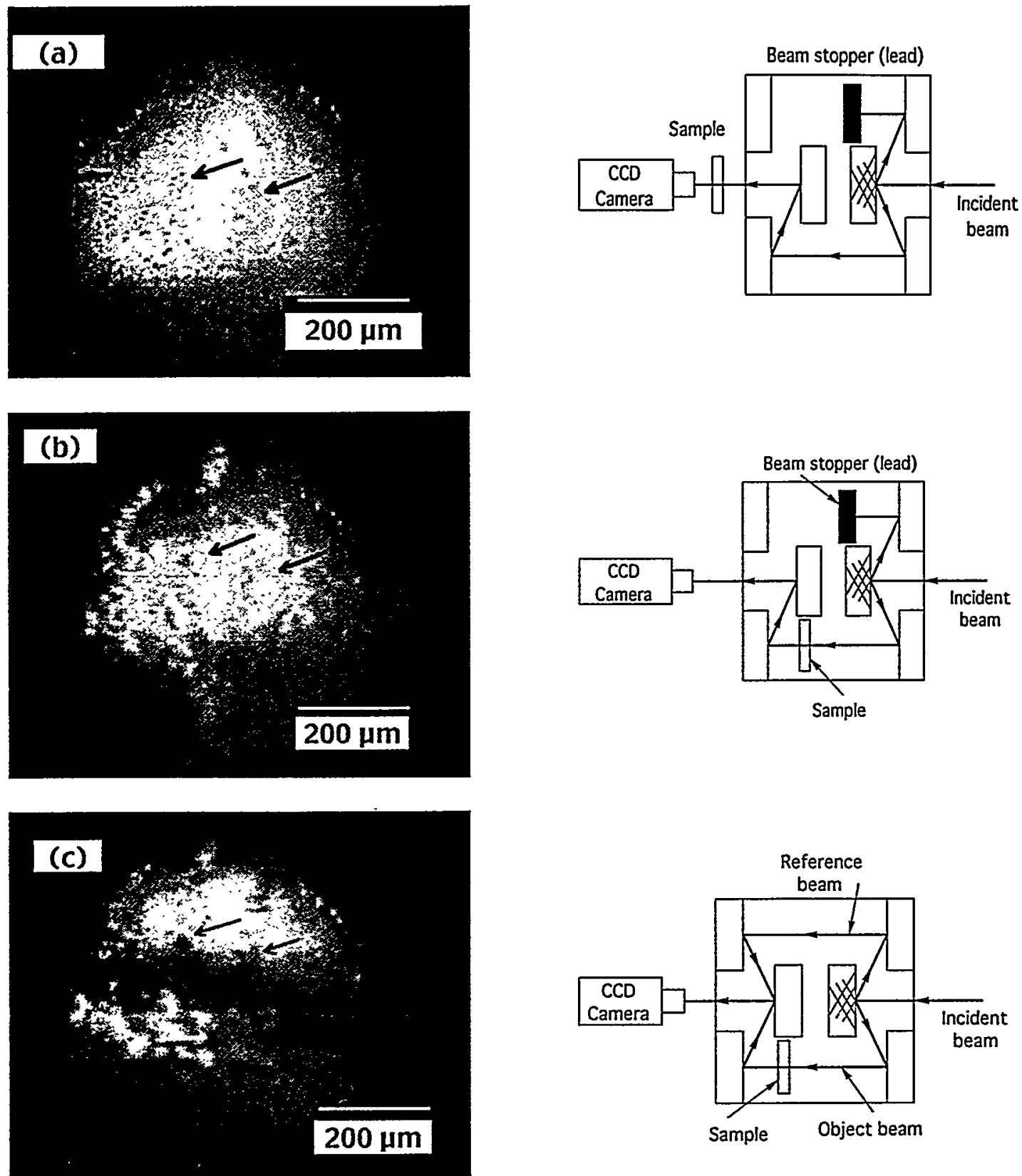

FIGURE 4. Phase contrast images of animal cancer cells deposited a thin plastic film. (a) Fresnel imaging and setup. (b) Angular-resolved imaging and setup (c) Holographic imaging and setup.

The third type of phase contrast image (Fig. 4c) corresponds to a pure phase or a holographic image, where interference occurs between an object and a reference beam. When a point-to-point interference occurs between an object wave going 
through the sample and a reference wave, the contrast is due only to the local optical thickness of the sample. The dark horizontal strip in this image is a Moiré fringe. Unlike the previous two techniques, this method of phase contrast is sensitive to all phase changes and not just to sharp phase jumps (edge detection). Thus, smooth changes in phase can be detected. Although the quality of the above images is not great, they provide a qualitative comparison of the different methods of phase contrast imaging.

\section{MEASUREMENTS AT $14.91 \mathrm{keV}-\mathrm{Si}(880) / \mathrm{Si}(808)$}

Although this interferometer was originally designed to work at $7.46 \mathrm{keV}$ using $\mathrm{Si}(440) / \mathrm{Si}(404)$ reflections, it is sometimes useful to be able to work at a higher energy. The ratio between the sensitivity of phase contrast and absorption is proportional to the $\mathrm{X}$-ray energy, and beam-path absorption (air) losses are smaller for higher energy. Thus, we proceeded to test the interferometer at $14.91 \mathrm{keV}$, using the $\mathrm{Si}(880) / \mathrm{Si}(808)$ reflections.
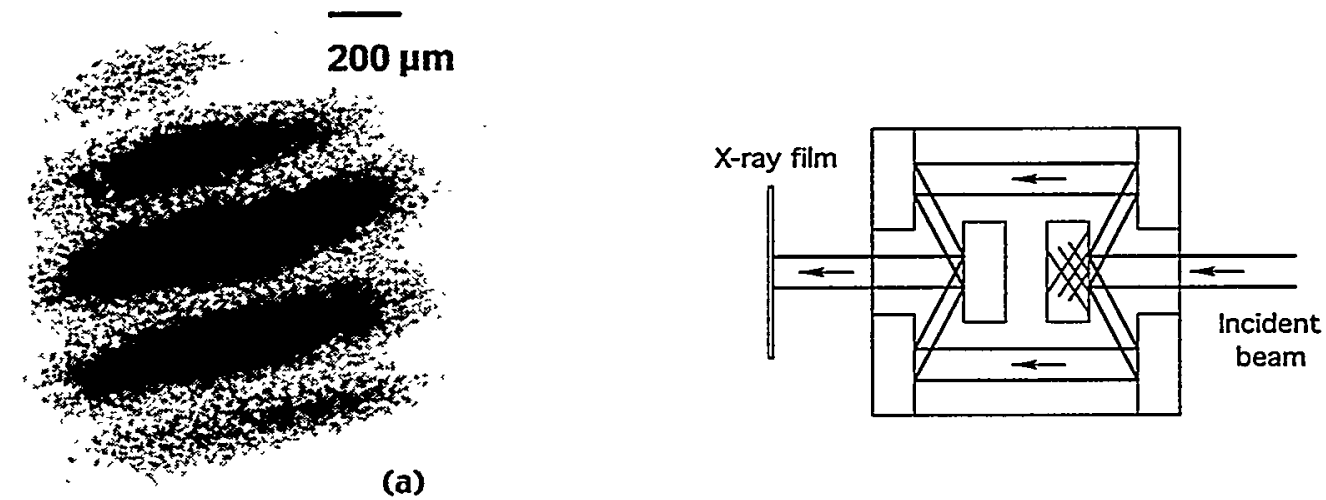

(a)
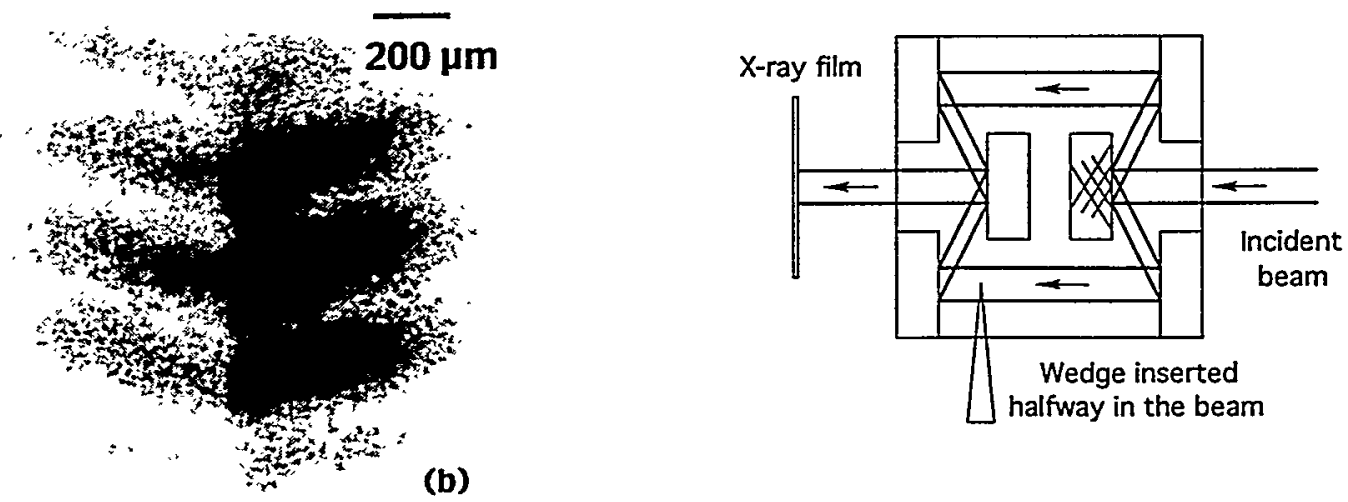

FIGURE 5. (a) Crystal Moire Fringes at $15 \mathrm{keV}$. (b) The same Moiré fringes with a plastic wedge halfway into one beam. 
At this higher order reflection, the interferometer spectral transmission is about $\Delta \lambda / \lambda \sim 2 \times 10^{-7}$. For an incoming photon count rate $(14.91 \mathrm{keV})$ of about $10^{13} \mathrm{ph} / \mathrm{s}$, the transmitted count rate was about $10^{7} \mathrm{ph} / \mathrm{s}$. Figure 7 a shows a Moire pattern at 14.91 $\mathrm{keV}$, from the $\mathrm{Si}(880) / \mathrm{Si}(808)$ reflections using dental film. In Figure $5 \mathrm{~b}$, we inserted an $8^{\circ}$ plastic wedge to cover half of one of the arms of the interferometer. The resulting effect of this linear phase gradient is a tilt of the fringes, which confirms that Moire pattern is indeed a two-beam interference effect. No fringes were seen with one arm of the interferometer blocked.

\section{CONCLUSION}

The use of only external Bragg-reflected beams, like in the BBBB interferometer, allows for less absorption loss and higher spatial resolution than the transmission setups (useful for imaging). We have started using asymmetric crystals to enlarge the usable beam size over several millimeters $(\sim 10 \mathrm{~mm})$ for large samples. High contrast Moire patterns over the whole expanded beam have been obtained. With new crystals and design, we hope to reduce the residual strain of the interferometer so as to avoid the Moiré pattern and obtain a clean and flat field of view.

\section{ACKNOWLEDGEMENTS}

We would like to thank $Z$. Cai from SRI-Cat (APS/ANL), for lending us his CCD camera. This work has been supported by the U.S. Department of Energy, BESMaterials Science under contract no. W-31-109-ENG-38.

\section{REFERENCES}

\footnotetext{
' D. G. Chetwynd et al., Nanotechnology 9 (1998) 125-132.

${ }^{2}$ A. Momose, NIM A352 (1995) 622-628.

${ }^{3}$ W. Graeff, U. Bonse, Z. Physik B27 (1977) 19.

${ }^{4}$ P. Cloetens et al., J. Phys. D29 (1996) 133-146.

${ }^{5}$ T. J. Davis et al., PRL 74 (1995) 3173.

${ }^{6} \mathrm{M}$. Born and E. Wolf, Principles of Optics, $2^{\text {nd }}$ edn. (Pergamon, Oxford, 1964).
} 